\title{
Cancer and alterations in the endocytic pathway
}

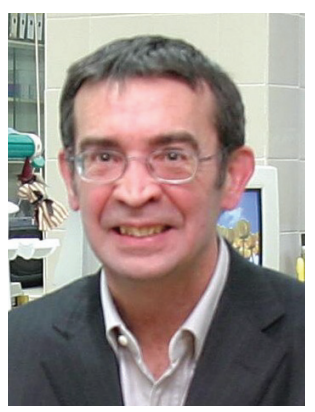

Rafael Rosell Catalan Institute of Oncology, Hospital Germans Trias i Pujol, Ctra Canyet, s/n, 08916 Badalona (Barcelona), Spain Tel.: +34934978925; Fax: +34934978950; rrosell@ico.scs.es

\author{
'Alterations that uncouple RTKs \\ (EGFR) from c-Cbl-mediated \\ ubiquitination, therefore causing \\ downregulation, are tightly \\ associated with the \\ pathogenesis of cancer...'
}

To date, translational research in cancer has focused on traditional pathways that regulate cell division, DNA repair, apoptosis, mutations, oncogenes and tumor suppressor genes. However, alterations in proteins of the endocytic pathway have recently been associated with tumors. Disrupted regulation of the endocytic pathway can concomitantly disrupt several different signaling pathways to affect growth, differentiation and survival [1]. Increased receptor tyrosine kinase (RTK) signaling in lung cancer is often caused by gene amplification, increased transcription/translation, or mutations that promote ligand-independent autophosphorylation. The failure of RTKs to be appropriately deactivated can cause neoplastic growth and resistance to the EGF receptor (EGFR) tyrosine kinase inhibitors (TKIs). A major deactivation pathway for RTKs, termed receptor downregulation, involves their ligandinduced internalization by means of endocytosis, followed by degradation in lysosomes.

\section{EGFR endocytosis \& $\mathrm{c}-\mathrm{Cbl}$}

Binding of EGF to the EGFR results in dimerization and autophosphorylation of several tyrosine residues in the cytoplasmic tail of the receptor. One of the phosphorylated tyrosines provides a binding site for the $\mathrm{SH} 2$ domain of the E3 ubiquitin ligase, $\mathrm{c}-\mathrm{Cbl}$ [2]. In turn, $\mathrm{c}-\mathrm{Cbl}$ is phosphorylated, thereby activating its ubiquitin ligase ability. Next to the $\mathrm{SH} 2$ domain of c-Cbl is a RING-finger domain that recruits a ubiquitinloaded E2 enzyme, and this machinery covalently tags EGFR with ubiquitin. EGFR is modified by Cbl-mediated mono-ubiquitination for endocytosis and degradation of the receptor. The adaptor protein, CIN85, interacts and functions with $\mathrm{c}-\mathrm{Cbl}$ in EGFR downregulation. Additionally, CIN85 interacts with a regulatory component of clathrin-coated pits, endophilin. Both EGFR and the hepatocyte growth factor receptor, Met, depend on the assembly of a c-Cbl/CIN85/endophilin complex at the active receptors in order to accelerate endocytosis and thereby attenuate signaling activity [2]. Alterations that uncouple RTKs (EGFR) from c-Cbl-mediated ubiquitination, therefore causing downregulation, are tightly associated with the pathogenesis of cancer and resistance to EGFR TKIs.

In addition, Met, colony-stimulating factor (CSF)-1 receptor (CSF-1R), PDGF receptor and EGFR are substrates for ubiquitination and depend on the interaction with c-Cbl for proper degradation [3]. Recently, Met amplification has been detected in $22 \%$ of non-small-cell lung cancer (NSCLC) specimens with EGFR mutations that became resistant to gefitinib or erlotinib [4]. Activation of c-Met results in the binding and phosphorylation of adaptor proteins such as $\mathrm{c}-\mathrm{Cbl}$ [5].

'A translocation between the genes encoding HIPI and the PDGF- $\beta$ receptor results in the expression of a HIP1-PDGF $\beta$ receptor fusion protein in chronic myelomonocytic leukemia.'

\section{EGFR Y $1045 \mathrm{~F}$ mutant induces defective endocytosis}

EGFR mutants lacking only the direct c-Cbl binding site elicit stronger mitogenic signals than the wild-type receptor. Replacement of Tyr1045 in EGFR with a phenylalanine $(\mathrm{Y} 1045 \mathrm{~F})$ reduces ligand-induced downregulation in living cells and significantly reduces receptor ubiquitylation [6]. Thus, a c-Cbl's site mutant receptor $(\mathrm{Y} 1045 \mathrm{~F})$ with defective endocytosis and increased recycling is endowed with enhanced signaling capacity.

\section{Huntingtin-interacting protein 1}

Huntingtin-interacting protein 1 (HIP1), which interacts with actin-binding proteins, is considered the first component of the endocytosis machinery, playing a fundamental role in 
clathrin trafficking. HIP1 is mutated in Huntington's disease [1]. Overexpression of HIP1 has been observed in breast cancer. The increased EGFR levels observed in breast cancers (basallike type) might be partially explained by increased HIP1 expression, which leads to reduced EGFR downregulation [3]. Overexpression of HIP1 might prevent EGFR endocytosis, which may minimize the effect of EGFR TKIs in breast cancer. Therefore, HIP1 plays an important role in receptor trafficking and tumorigenesis. Binding of a growth factor to its receptor results in endocytosis of the ligand-receptor complex. Accessory endocytic proteins, including HIP1, mediate the formation of clathrincoated pits from the plasma membrane. These pits then convert to clathrin-coated vesicles and early endosomes [1].

\section{'...increased EGFR levels observed \\ in breast cancers (basal-like type) might be partially explained by increased HIPI expression...}

A translocation between the genes encoding HIP1 and the PDGF- $\beta$ receptor results in the expression of a HIP1-PDGF- $\beta$ receptor fusion protein in chronic myelomonocytic leukemia [7]. Several tumors, including breast, colon, kidney, lung, melanoma, ovarian and prostate tumors, have high levels of HIP1 protein. This pattern has been confirmed by analysis of HIP1 expression using immunohistochemical staining of primary human cancer tissue microarrays, which demonstrated moderate to high staining of HIP1 in most of these types of cancer [8]. In prostate cancer, patients with HIP1-negative tumors did not relapse after radical prostatectomy, whereas $28 \%$ of those with HIP1-positive tumors did [8]. For breast tumors, elevated levels of HIP1 expression have been observed in a typical ductal hyperplasia, ductal carcinoma in situ and invasive breast cancer [9].

\section{Other mechanisms of $\mathrm{c}-\mathrm{Cbl}$ inactivation}

EGFR can avoid lysosomal downregulation through the loss of the c-Cbl-binding site, inefficient $\mathrm{c}$-Cbl recruitment, or the formation of fusion proteins that escape endocytosis and the degradative lysosomal pathway. Mechanisms of c-Cbl inactivation include:

- Degradation of both EGFR and Cbl: EGF induces a loss of $\mathrm{Cbl}$ protein that parallels a decrease in EGFR [10];
- Degradation of both KIT and Cbl: stem cell factor, the ligand for KIT, induces the interaction between KIT and $\mathrm{Cbl}$ proteins and their mutual degradation [11];

- c-Cbl ubiquitinates CSF-1: addition of CSF-1 to macrophages stimulates rapid transient tyrosine phosphorylation, membrane association and multi-ubiquitination of $\mathrm{Cbl}[12,13]$;

- Degradation of c-Cbl by Src: co-overexpression of Src and EGFR characterizes relatively aggressive animal tumors. In response to the activation of c-Src, c-Cbl proteins undergo tyrosine phosphorylation that promotes their ubiquitylation and proteasomal destruction. By promoting the destruction of c-Cbl, c-Src enables EGFR to evade desensitization [14];

- Degradation of Cbl proteins by HECT E3 ligases: HECT E3 ubiquitinates several proteins including IGF-1 and Cbl [15].

- Interaction with T-cell ubiquitin ligand (TULA): the UBA- and SH3-containing protein TULA was identified as a c-Cbl-associated protein. TULA mediates inhibition of the effects of $\mathrm{c}-\mathrm{Cbl}$ on protein tyrosine kinases. TULA induces the ubiquitylation and degradation of c-Cbl [16]. TULA is also known as Sts- 1 and $-2[15,17]$;

- Inhibition of Cbl by Sprouty: Sprouty 2 or 1 forms a trimolecular complex with c-Cbl and CIN85 that is required for the inhibition of c-Cbl-mediated EGFR degradation. Thus, Sprouty 2 functions as a positive regulator of EGFR function. Overexpression of Sprouty 2 could contribute to carcinogenesis by preventing Cbl-mediated downregulation of EGFR [15];

- Inhibition of Cbl by Cdc42: Cdc42 is a rasrelated protein that can be activated by several extracellular stimulants, including EGF. Activation of Cdc42 protects EGFR from the negative regulatory activity of the $\mathrm{c}-\mathrm{Cbl}$ ubiquitin ligase. Activated $\mathrm{Cdc} 42$ binds to p85 cloned-out-of-library (Cool)-1/ $\beta$-Pak-interactive exchange factor (Pix), a protein that directly associates with c-Cbl. This inhibits the binding of $\mathrm{Cbl}$ by EGFR and thus prevents $\mathrm{Cbl}$ from catalyzing receptor ubiquitination [18];

- Cortactin: overexpression of cortactin prevents downregulation of EGFR. Cortactin is a multidomain protein that associates with the actin-related protein (Arp)2/3 and regulates actin polymerization [19]. Expression of Arp2/3 is increased in colon cancer [20]. Overexpression of cortactin has been found in breast and head-and-neck cancers [15]; 
- Others: other mechanisms of abrogating c-Cbl function involve the proteins Alix, HPV16 E5 and ESCRT-I [15].

\section{Conclusion}

To date, more than 150 proteins have been reported to be affected by $\mathrm{Cbl}$. Cbl-associated proteins have been identified by several methods, including pulldown assays, immunofluorescence, immunoprecipitation and yeast two-hybrid screens. The list of Cbl-interacting partners is not yet completed [21]. In summary, we can infer that $\mathrm{Cbl}$ function is impaired in many tumors, and a greater understanding of the mechanisms of this dysfunction in the endocytic pathway could have important clinical consequences for prognosis and for predicting response or resistance to targeted therapies with TKIs. Research is warranted, particularly on novel mechanisms of resistance to EGFR TKIs in NSCLC patients with wild-type or mutated EGFR.

\section{Financial \& competing interests disclosure}

The authors have no relevant affiliations or financial involvement with any organization or entity with a financial interest in or financial conflict with the subject matter or materials discussed in the manuscript. This includes employment, consultancies, honoraria, stock ownership or options, expert testimony, grants or patents received or pending, or royalties.

No writing assistance was utilized in the production of this manuscript.

\section{Bibliography}

Papers of special note have been highlighted as either of interest $(\bullet)$ or of considerable interest $(\bullet \bullet)$ to readers.

1. Hyun TS, Ross TS: HIP1: trafficking roles and regulation of tumorigenesis. Trends Mol. Med. 10, 194-199 (2004).

2. Thien CB, Langdon WY: Negative regulation of PTK signalling by $\mathrm{Cb}$ proteins. Growth Factors 23, 161-167 (2005).

-• Outstanding review of $\mathrm{Cbl}$ functioning in the tyrosine kinase signaling pathway.

3. Bache KG, Slagsvold T, Stenmark H: Defective downregulation of receptor tyrosine kinases in cancer. $E M B O J .23$, 2707-2712 (2004).

4. Engelman JA, Zejnullahu K, Mitsudomi T et al:: MET amplification leads to gefitinib resistance in lung cancer by activating ERBB3 signaling. Science 316, 1039-1043 (2007).

-• Recent new mechanism of resistance to gefitinib in patients with EGF receptor mutations by Met amplification.

5. Christensen JG, Schreck R, Burrows J et al: : A selective small molecule inhibitor of c-Met kinase inhibits c-Met-dependent phenotypes in vitro and exhibits cytoreductive antitumor activity in vivo. Cancer Res. 63, 7345-7355 (2003).

6. Waterman $\mathrm{H}$, Katz M, Rubin C et al:: A mutant EGF-receptor defective in ubiquitylation and endocytosis unveils a role for Grb2 in negative signaling. EMBO J. 21, 303-313 (2002).

7. Ross TS, Bernard OA, Berger R et al:: Fusion of Huntingtin interacting protein 1 to plateletderived growth factor beta receptor (PDGF- $\beta$ R) in chronic myelomonocytic leukemia with $\mathrm{t}(5 ; 7)(\mathrm{q} 33 ; \mathrm{q} 11.2)$. Blood 91 , 4419-4426 (1998).
8. Rao DS, Hyun TS, Kumar PD et al: Huntingtin-interacting protein 1 is overexpressed in prostate and colon cancer and is critical for cellular survival. J. Clin. Invest. 110, 351-360 (2002).

9. Rao DS, Bradley SV, Kumar PD et al: Altered receptor trafficking in Huntingtin interacting protein 1-transformed cells. Cancer Cell 3, 471-482 (2003).

10. Ettenberg SA, Magnifico A, Cuello $\mathrm{M}$ et al: : Cbl-b-dependent coordinated degradation of the epidermal growth factor receptor signaling complex. J. Biol. Chem. 276, 27677-27684 (2001)

11. Zeng S, Xu Z, Lipkowitz S et al:: Regulation of stem cell factor receptor signaling by $\mathrm{Cbl}$ family proteins (Cbl-b/c-Cbl). Blood 105, 226-232 (2005).

12. Wang Y, Yeung YG, Langdon WY et al.: c-Cbl is transiently tyrosine-phosphorylated, ubiquitinated, and membrane-targeted following CSF-1 stimulation of macrophages. J. Biol. Chem. 271, 17-20 (1996).

13. Wang Y, Yeung YG, Stanley ER: CSF-1 stimulated multiubiquitination of the CSF-1 receptor and of Cbl follows their tyrosine phosphorylation and association with other signaling proteins. J. Cell Biochem. 72, 119-134 (1999).

14. Bao J, Gur G, Yarden Y: Src promotes destruction of c-Cbl: implications for oncogenic synergy between Src and growth factor receptors. Proc. Natl Acad. Sci. USA 100, 2438-2443 (2003).

15. Ryan PE, Davies GC, Nau MM et al: Regulating the regulator: negative regulation of Cbl ubiquitin ligases. Trends Biochem. Sci. 31, 79-88 (2006).
-. Comprehensive review of the numerous mechanisms that inhibit Cbl function.

16. Feshchenko EA, Smirnova EV, Swaminathan G et al: TULA: an SH3- and UBA-containing protein that binds to c-Cbl and ubiquitin. Oncogene 23, 4690-4706 (2004).

17. Kowanetz K, Crosetto N, Haglund K et al:: Suppressors of T-cell receptor signaling Sts- 1 and Sts- 2 bind to $\mathrm{Cbl}$ and inhibit endocytosis of receptor tyrosine kinases. J. Biol. Chem. 279, 32786-32795 (2004).

18. Wu WJ, Tu S, Cerione RA: Activated Cdc42 sequesters c-Cbl and prevents EGF receptor degradation. Cell 114, 715-725 (2003).

19. Daly RJ: Cortactin signalling and dynamic actin networks. Biochem. J. 382, 13-25 (2004).

20. Otsubo T, Iwaya K, Mukai $Y$ et al.: Involvement of Arp2/3 complex in the process of colorectal carcinogenesis. Mod. Pathol. 17, 461-467 (2004).

21. Schmidt MH, Dikic I: The Cbl interactome and its functions. Nat. Rev. Mol. Cell Biol. 6, 907-919 (2005).

\section{Affiliation}

- Rafael Rosell, MD, Chief, Medical Oncology Service \& Scientific Director of Oncology Research Catalan Institute of Oncology, Hospital Germans Trias i Pujol, Ctra Canyet, s/n, 08916 Badalona (Barcelona), Spain Tel.: +34934978925; Fax: +34934978950; rrosell@ico.scs.es 